\title{
Is there life in the formalin fixed neutrophil for ANCA testing? $\mathrm{NO}$ !
}

Antineutrophil cytoplasmic antibodies (ANCA) represent a family of autoantibodies directed against various neutrophil proteins, principally enzymes. These antibodies have been described in association with a number of autoimmune or inflammatory conditions. Initial high sensitivity and specificity for necrotising vasculitic conditions such as Wegener's granulomatosis and microscopic polyarteritis were reported, but as these tests became more widely available and were applied to a broader range of unwell patients, performance criteria inevitably fell. Initially ANCA were detected on ethanol fixed neutrophils at a time when the target antigens for the fluorescent patterns had not yet been defined, and two major patterns of fluorescence were identified. The cytoplasmic (C-ANCA) pattern was later shown to be principally associated with sera showing antibody activity against the neutrophil proteinase 3 (PR3), whereas the perinuclear (P-ANCA) was associated with activity against various neutrophil enzymes of which myeloperoxidase (MPO) was the most frequent and important. Technically the detection of P-ANCA by immunofluorescence caused more problems, particularly since it was difficult to differentiate the pattern from the staining shown by antinuclear or granulocyte specific nuclear antibodies, which occurs in many of the sera from patients with other vasculitic conditions under investigation.

To circumvent this problem, several laboratories incorporated a protocol in which formalin fixed neutrophils were included since it was claimed that this form of fixation prevented the redistribution of cytoplasmic enzymes while retaining their reactivity, allowing a differentiation between
P-ANCA and antinuclear antibodies. This approach received endorsement by the Association of Clinical Pathologists ${ }^{1}$ and has been widely adopted in the United Kingdom but not elsewhere.

Now that there are solid phase ELISA based enzyme specific assays available for confirmation, it is time for the whole approach to ANCA testing to be reassessed and to a large extent this debate was opened up in a previous editorial by Spickett and Broomhead. ${ }^{2}$ These investigators now present evidence in a paper in this issue ${ }^{3}$ that clearly indicates that formalin neutrophil fixation by a variety of methods does not adequately differentiate between antinuclear antibody fluorescence and MPO associated P-ANCA staining, and may cause diagnostic confusion.

This is a major concern in view of the wide current availability of ANCA testing (134 laboratories in UKNEQAS) and its increasing application to patients in whom there is a low pretest probability of ANCA associated disease. In this patient group, in whom antinuclear antibodies are frequently encountered, the issue of a false positive P-ANCA report based on formalin fixation appearances may result in inappropriate further investigation, classification, and, most dangerously of all, treatment. Its use should now be abandoned.

Fortunately international consensus and standardisation points to a more appropriate approach. A recently published article and accompanying editorial ${ }^{4}$ indicate $^{2}$ high performance characteristics when immunofluorescence screening combined with enzyme specific ELISA confirmation is employed on patients with a robust diagnosis of clinical or histopathological systemic vasculi-

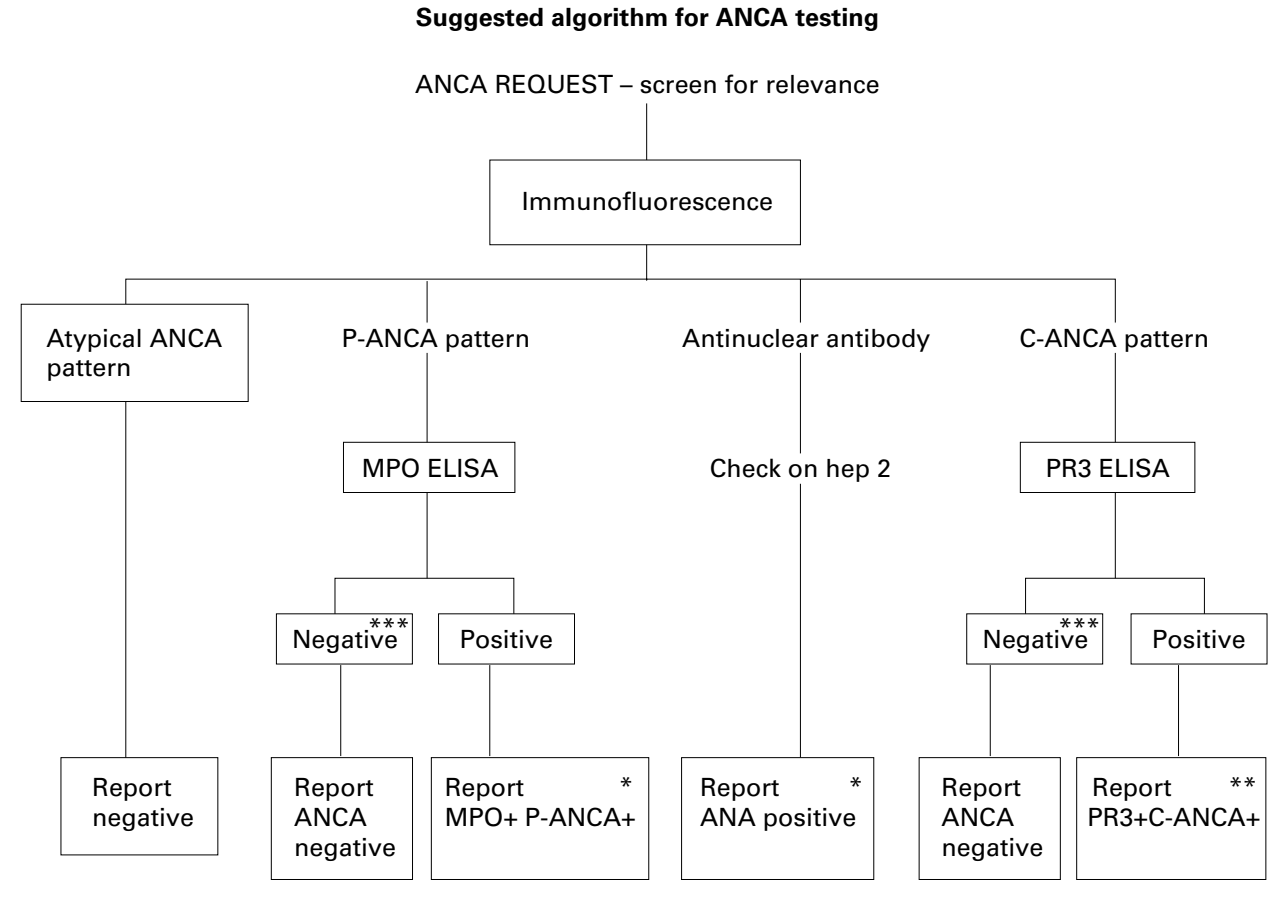

* Sensitivity $49.9 \%$, specificity $99 \%$ in microscopic polyarteritis, $46 \% / 99 \%$ in Wegener's granulomatosis (4). * Sensitivity $57 \%$, specificity $99 \%$ in Wegener's granulomatosis (4).

***Check alternative ELISA if high probability of vasculitis.

Figure 1 Suggested algorithm for ANCA testing. 
tis, when compared with relevant disease controls. The use of enzyme specific ELISA alone will not improve specificity without loss of sensitivity, particularly since some sera from disease control patients give non-specific false positive ELISA results. However, combining immunofluorescence with ELISA gives greatly improved specificity with only minor loss of sensitivity. Laboratories should remember that some cases of negative C- or P-ANCA occur even in cases of biopsy proven rapidly progressive pauci-immune glomerulonephritis or Wegener's, and that biopsy remains the gold standard investigation. However, negative C- and P-ANCAs using these combinations of tests are valuable in ruling out ANCA associated systemic vasculitis in low likelihood cases, providing that the laboratory is skilled in immunofluorescence interpretation.

As Spickett and Broomhead indicate, ${ }^{2}$ interpretation of immunofluorescent patterns requires skill. This can demonstrably be achieved at national reference centres with high throughput ${ }^{4}$ but, on the basis of recent UK-NEQAS returns, not by a large proportion of UK participating laboratories. Replacing fluorescence with ELISA alone will not improve the situation as false positive results will again occur.

On the basis of evidence, laboratories should cease formalin testing, screen on ethanol fixed neutrophils only, and confirm positive results with enzyme specific MPO or PR3 ELISA assays. Clinicians must be made aware of the fact that these tests only perform well in clinical situations where the pretest probability of systemic vasculitis is high, and they should be discouraged from indiscriminate use of ANCA testing in patients in whom non-specific malaise, pyrexia, or raised ESR of unknown origin are the presenting complaints. In such situations, these tests will continue to perform poorly.

A proposed algorithm for ANCA testing is given in fig 1 .

A G BIRD

Department of Immunology, Oxford Radcliffe Hospital Trust, Churchill Hospital, Headington, Oxford OX3 7LF, UK

1 Lock RJ. Detection of autoantibodies to neutrophil cytoplasmic antigens. $\mathcal{F}$ Clin Pathol 1994;47:4-8.

2 Spickett GP, Broomhead V. How to test for antineutrophil cytoplasmic antibody - evidence based immunology. F Clin Pathol 1999;52:81-2.

3 Chowdhury SMZ, Broomhead V, Spickett GP, et al. Pitfalls of formalin fixation for determination of antineutrophil cytoplasmic antibodies. $f$ Clin Pathol 1999;52:475-7.

4 Hagen EC, Daha MR, Hermans J, et al. Diagnostic value of standardised assays for anti-neutrophil cytoplasmic antibodies in idiopathic systemic vasculitis. Kidney Int 1998;53:743-53.

5 Jeanette JC, Wilksman AY, Falk RJ. Editorial. Diagnostic predictive value of ANCA serology. Kidney Int 1998;53:796-8. 